\title{
Identification of Ferroptosis-Related Genes in Alzheimer's Disease Based on Bioinformatic Analysis
}

\author{
Ying Wangt, Guohua Chen and Wei Shao*t
}

Department of Neurology, Wuhan Hospital of Traditional Chinese and Western Medicine, Tongji Medical College, Huazhong University of Science and Technology, Wuhan, China

Introduction: Alzheimer's disease (AD) is the most prevalent cause of dementia, and emerging evidence suggests that ferroptosis is involved in the pathological process of $A D$.

Materials and Methods: Three microarray datasets (GSE122063, GSE37263, and GSE140829) about AD were collected from the GEO database. AD-related module genes were identified through a weighted gene co-expression network analysis (WGCNA). The ferroptosis-related genes were extracted from FerrDb. The apoptosis-

OPEN ACCESS

Edited by:

Guiyou Liu,

Tianjin Institute of Industrial Biotechnology, Chinese Academy

of Sciences (CAS), China

Reviewed by:

Zhihao Wu,

Southern Methodist University,

United States

Anandhan Annadurai,

University of Arizona, United States

*Correspondence:

Wei Shao

shaowei74@126.com

tThese authors have contributed

equally to this work

Specialty section:

This article was submitted to

Neurogenomics,

a section of the journal

Frontiers in Neuroscience

Received: 28 November 2021

Accepted: 06 January 2022

Published: 07 February 2022

Citation:

Wang $Y$, Chen $G$ and Shao W

(2022) Identification

of Ferroptosis-Related Genes in Alzheimer's Disease Based on

Bioinformatic Analysis.

Front. Neurosci. 16:823741. doi: 10.3389/fnins.2022.823741 related genes were downloaded from UniProt as a control to show the specificity of ferroptosis. The overlap was performed to obtain the module genes associated with ferroptosis and apoptosis. Then the Gene Ontology (GO) and Kyoto Encyclopedia of Genes and Genomes (KEGG) pathway enrichment analyses and the protein-protein interaction (PPI) were conducted. Cytoscape with CytoHubba was used to identify the hub genes, and the Logistic regression was performed to distinguish the $A D$ patients from controls.

Results: 53 ferroptosis-related module genes were obtained. The GO analysis revealed that response to oxidative stress and starvation, and multicellular organismal homeostasis were the most highly enriched terms. The KEGG analysis showed that these overlapped genes were enriched not only in renal cell carcinoma pathways and central carbon metabolism in cancer, but also in autophagy-related pathways and ferroptosis. Ferroptosis-related hub genes in AD (JUN, SLC2A1, TFRC, ALB, and NFE2L2) were finally identified, which could distinguish $A D$ patients from controls $(P<0.05)$. The area under the ROC curve (AUC) was 0.643. Apoptosis-related hub genes in AD (STAT1, MCL1, and BCL2L11) were also identified and also could distinguish $A D$ patients from controls $(P<0.05)$. The AUC was 0.608 , which was less than the former $A \cup C$ value, suggesting that ferroptosis was more special than apoptosis in AD.

Conclusion: We identified five hub genes (JUN, SLC2A1, TFRC, ALB, and NFE2L2) that are closely associated with ferroptosis in $A D$ and can differentiate $A D$ patients from controls. Three hub genes of apoptosis-related genes in AD (STAT1, MCL1, and BCL2L11) were also identified as a control to show the specificity of ferroptosis. JUN, SLC2A1, TFRC, ALB, and NFE2L2 are thus potential ferroptosis-related biomarkers for disease diagnosis and therapeutic monitoring.

Keywords: Alzheimer's disease (AD), WGCNA, ferroptosis, apoptosis, GEO 


\section{INTRODUCTION}

Alzheimer's disease (AD) is the most prevalent cause of dementia, accounting for approximately $60-80 \%$ of all cases (Gbd 2016

Dementia Collaborators, 2019). The exact pathogenesis of AD is still not fully elucidated (Zhang et al., 2021). Ferroptosis is an iron-dependent lipid peroxidation-driven cell death, and emerging evidence suggests that it is involved in the pathological process of AD (Lane et al., 2018; Weiland et al., 2019). In addition, several characteristics of the pathogenesis of $\mathrm{AD}$ were consistent

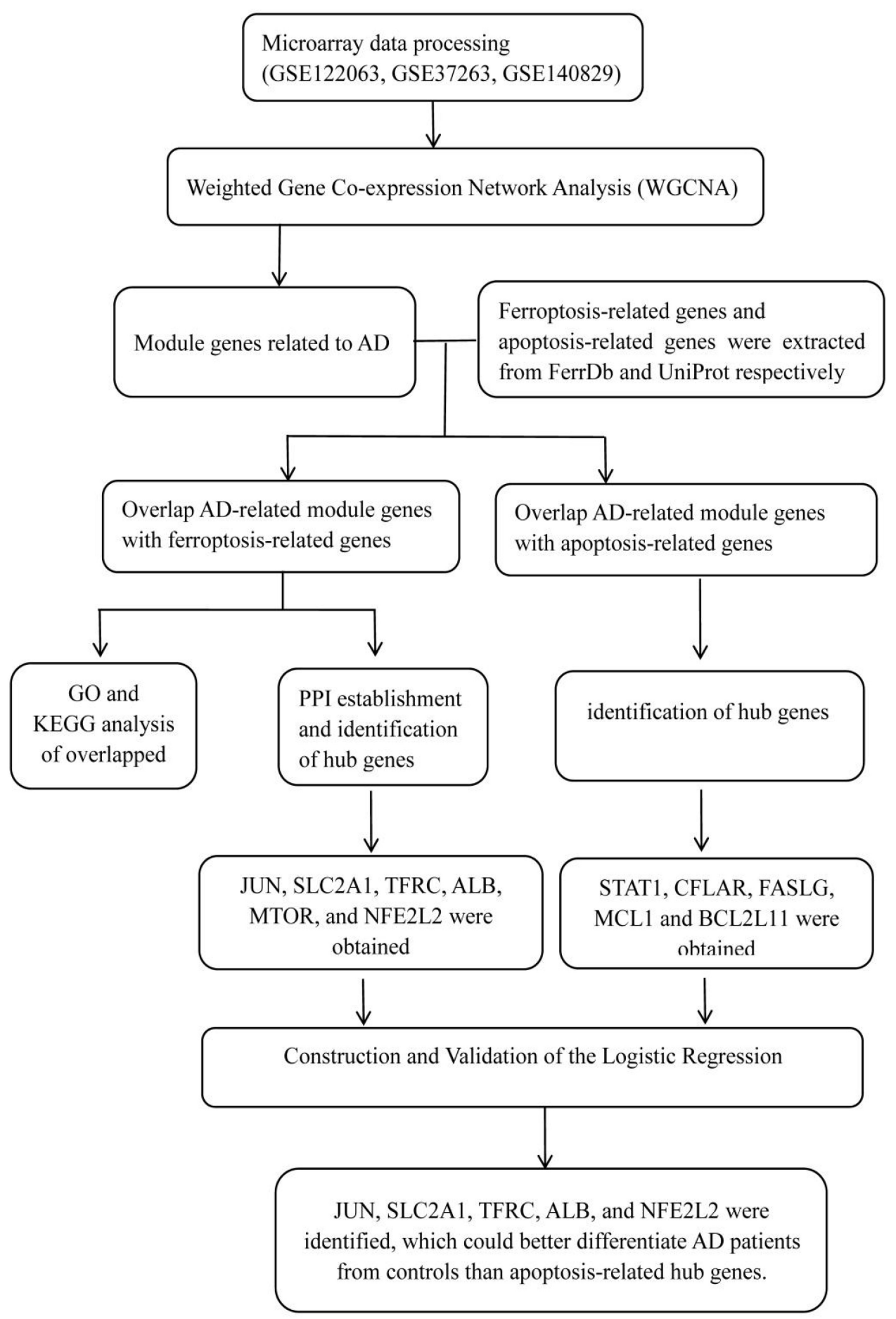

FIGURE 1 | The workflow chart of data preparation, processing, analysis, and validation. 
with those of ferroptosis, such as excess iron accumulation, elevated lipid peroxides (Zhang et al., 2012; Hambright et al., 2017; Ayton et al., 2019). Therefore, ferroptosis is increasingly being recognized as a unique cell death mechanism participating in the pathogenesis of $\mathrm{AD}$. However, more direct evidence is needed to be presented (Chen et al., 2021). Apoptosis is the
A

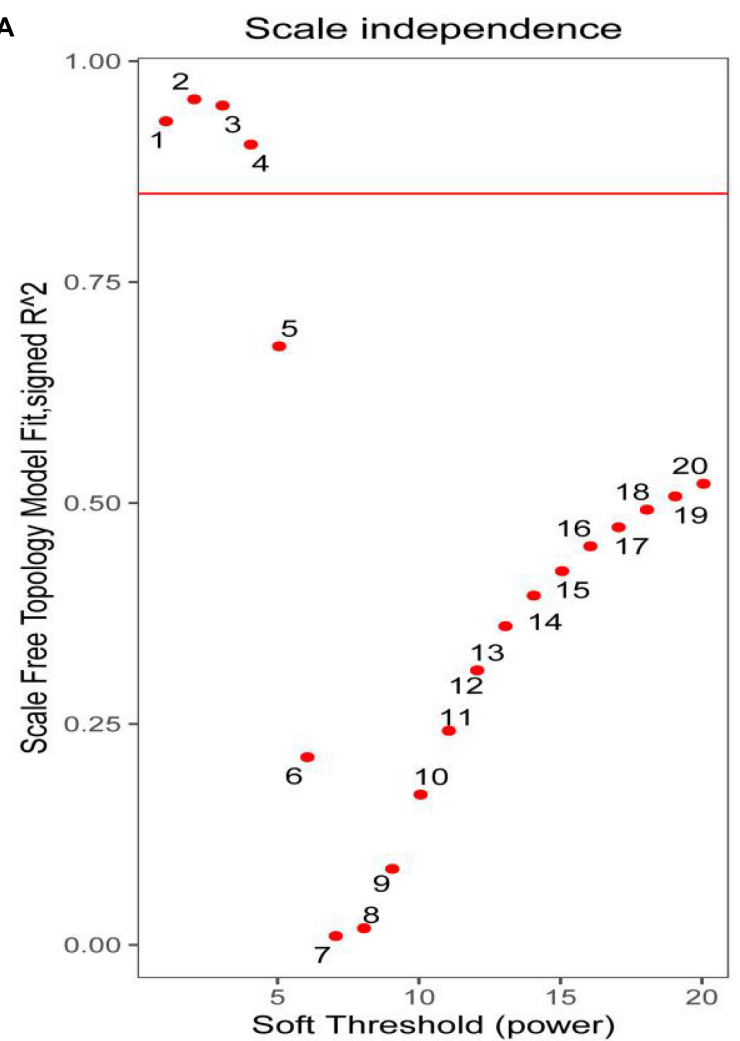

B

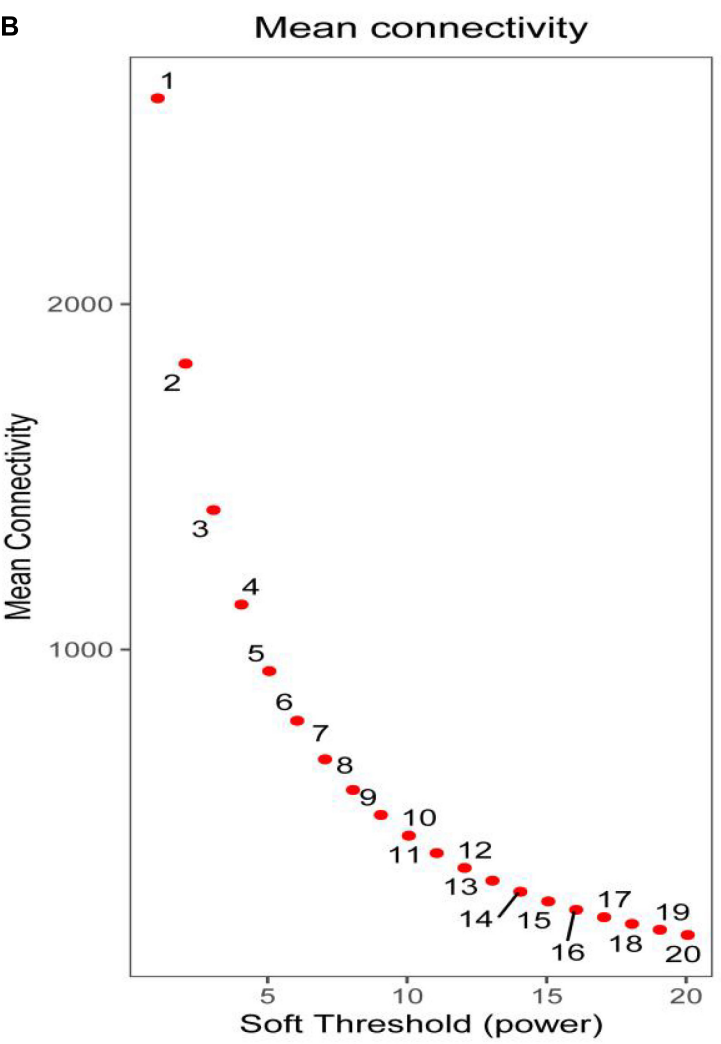

Soft Threshold (power)
C

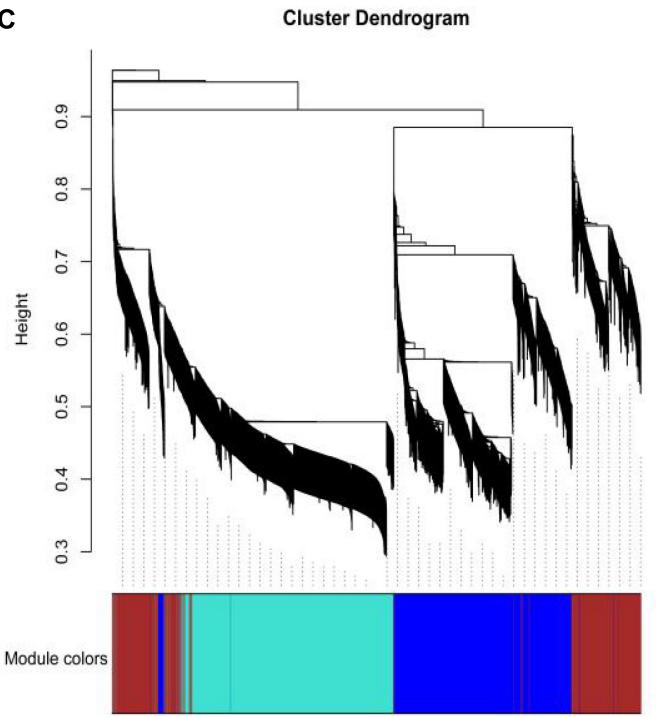

D

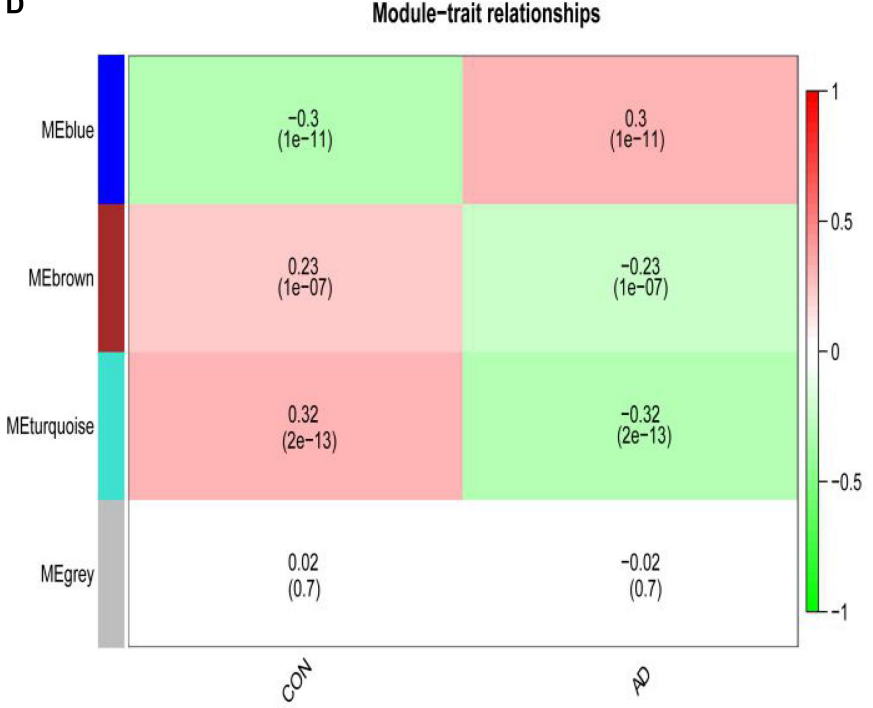

FIGURE 2 | (A) Analysis of the scale-free index for various soft-threshold powers (B). (B) Analysis of the mean connectivity for various soft-threshold powers. (C) Identification of co-expression gene modules. The branches of the dendrogram cluster into 4 modules and each one was labeled in a unique color. (D) A heatmap showing the correlation between each module eigengene and phenotype. Two modules were correlated with AD-namely, turquoise and blue modules. 
spontaneous and orderly death of cells, which involves the activation, expression and regulation of a series of genes, and it is a biological process that plays an essential role in normal physiology (Obulesu and Lakshmi, 2014). It is now generally accepted that massive neuronal death due to apoptosis is a common characteristic in the brains of patients suffering from neurodegenerative diseases, and apoptotic cell death has been found in neurons and glial cells in AD (Shimohama, 2000; Sharma et al., 2021).

Current studies on ferroptosis and $\mathrm{AD}$ are mainly focused on two aspects: one is the mechanism of ferroptosis in the pathological process of $\mathrm{AD}$, mainly discussing how ferroptosis participates in the AD (Masaldan et al., 2019; Jakaria et al., 2021); the second is the clinical efficacy study of ferroptosis inhibitors in $\mathrm{AD}$, mainly to explore whether ferroptosis as a drug target of $\mathrm{AD}$ can effectively delay the progression of AD (Yan and Zhang, 2019; Plascencia-Villa and Perry, 2021; Vitalakumar et al., 2021). The purpose of this study is to investigate the association between ferroptosis-related genes and $\mathrm{AD}$ with the gene level, which is a supplement to existing studies and also a reference for ferroptosis as a therapeutic target for $\mathrm{AD}$. These hub genes identified by this study could also serve as the ferroptosis-related biomarkers for disease diagnosis and therapeutic monitoring.

\section{MATERIALS AND METHODS}

\section{Microarray Data Processing}

Three microarray datasets (GSE122063, GSE37263, and GSE140829) of AD were collected from the GEO database ${ }^{1}$. GSE122063 was based on the platforms of the GPL16699 (Mckay et al., 2019); GSE37263 was based on the platforms of the GPL5175 (Tan et al., 2010); and GSE140829 was based on the platforms of the GPL15988. Data for $56 \mathrm{AD}$ patients and 44 control samples from GSE122063, 8 AD patients and 8 control

${ }^{1}$ http://www.ncbi.nlm.nih.gov/geo

TABLE 1 | Details for FerrDb.

\begin{tabular}{|c|c|c|c|c|}
\hline Data set & Category & Annotated from & Count & Annotations \\
\hline Driver & Regulator & Gene & 108 & 150 \\
\hline Suppressor & Regulator & Gene & 69 & 109 \\
\hline Marker & Marker & Gene & 111 & 123 \\
\hline Inducer & Regulator & Small molecule & 35 & 54 \\
\hline Inhibitor & Regulator & Small molecule & 41 & 46 \\
\hline Ferroptosis aggravates disease & Ferroptosis-diseaseassociation & Ferroptosis and disease & 49 & 58 \\
\hline Ferroptosis alleviates disease & Ferroptosis-diseaseassociation & Ferroptosis and disease & 46 & 77 \\
\hline
\end{tabular}

The number of "Count" and "Annotations" is inconsistent, because one gene can have multiple annotations.
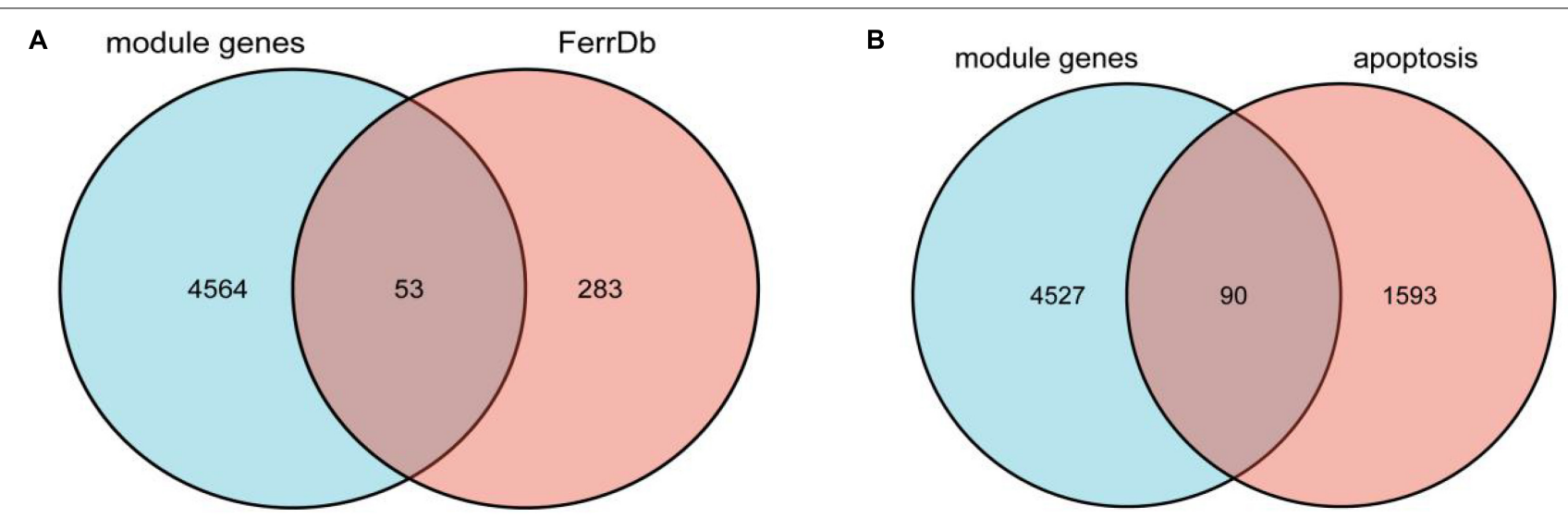

FIGURE 3 | (A) Venn diagram showing the numbers of overlapped genes between AD-related module genes and ferroptosis-related genes. (B) Venn diagram showing the numbers of overlapped genes between AD-related module genes and apoptosis-related genes.

TABLE 2 | Ferroptosis-related module genes obtained through the Venn diagram.

\begin{tabular}{ll}
\hline Type & Genes \\
\hline Driver & PGD, YY1AP1, ATG3, ATG7, DPP4, NRAS, LPIN1, FBXW7, SCP2, EPAS1, TF, ATG16L1, IDH1, TFRC, BAP1, SNX4, PIK3CA, ATF3, PRKAA2 \\
Suppressor & SQSTM1, SLC40A1, MTOR, FANCD2, MUC1, TP63, FTMT, PRDX6, NFE2L2, ACSL3, JUN, SLC7A11, FH, CISD2, SESN2, PROM2 \\
Marker & TXNIP, HSD17B11, NCF2, PTGS2, ALB, STEAP3, SLC1A4, RRM2, CXCL2, ANGPTL7, PRDX1, SLC2A1, STMN1, RGS4, OXSR1, KLHL24, \\
& CAPG, DRD5
\end{tabular}


samples from GSE37263, and $182 \mathrm{AD}$ patients and 207 control samples from GSE140829 were analyzed in our study. A flow diagram of the study is shown in Figure 1.

\section{Weighted Gene Co-expression Network Analysis}

Firstly, the expression profiles of three datasets were removed from the batch effect for further analysis. The gene co-expression network was constructed with an R package termed "weighted gene co-expression network analysis (WGCNA)" (Langfelder and Horvath, 2008, 2012). The Adjacency matrix was constructed by a weighted correlation coefficient. Subsequently, the adjacency matrix was transformed into a topological overlap matrix (TOM).
Then, hierarchical clustering was performed to identify modules, and the eigengene was calculated. Finally, we assessed the correlation between phenotype (i.e., AD or control samples) and each module by Pearson's correlation analysis and identified ADrelated modules. The genes in these modules were considered as AD-related module genes.

\section{The Extraction of Ferroptosis-Related Genes From FerrDb and Apoptosis-Related Genes From UniProt}

$\mathrm{FerrDb}^{2}$ is an artificial ferroptosis database for the management and identification of ferroptosis-related markers and regulatory

${ }^{2}$ http://www.zhounan.org/ferrdb

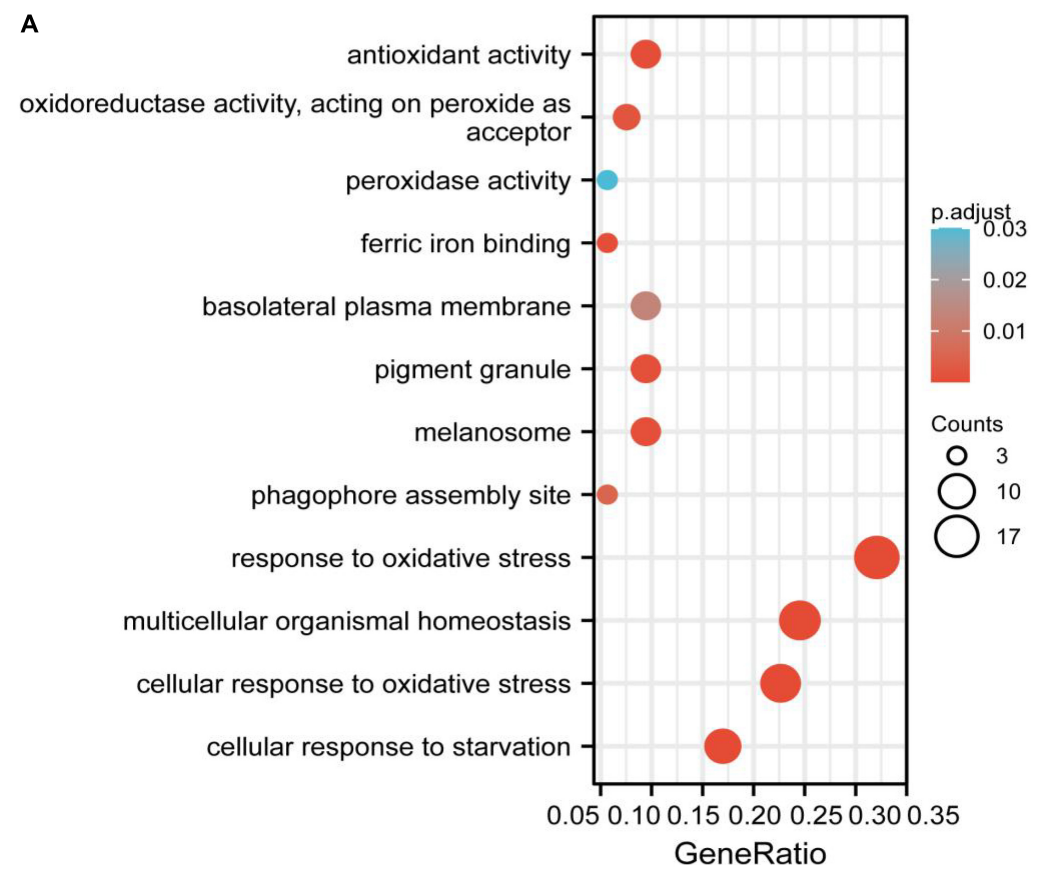

B

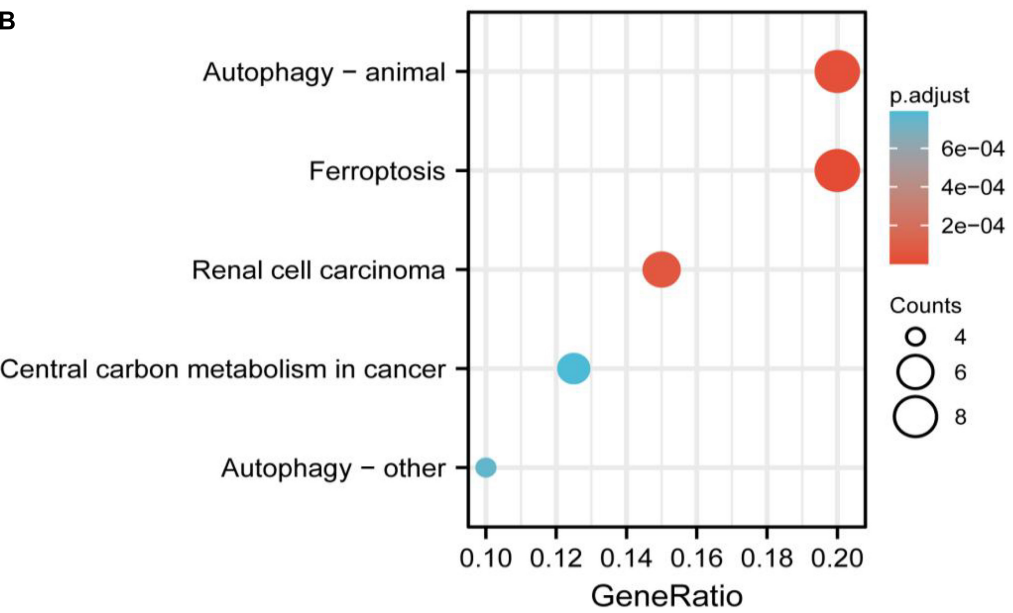

FIGURE 4 | (A) Gene Ontology (GO) functional analysis showing enrichment of ferroptosis-related module genes. (B) Kyoto Encyclopedia of Genes and Genomes (KEGG) pathway enrichment analysis of ferroptosis-related module genes. 
factors, as well as ferroptosis-related diseases (Zhou and Bao, 2020). Therefore, ferroptosis-related genes were downloaded from this database for further analysis. The UniProt Knowledgebase is the central hub for the collection of functional information on proteins, with accurate, consistent and rich annotation, and thus apoptosis-related genes were extracted from UniProt ${ }^{3}$.

${ }^{3}$ https://www.uniprot.org/

\section{Overlap Alzheimer's Disease-Related Module Genes With Ferroptosis-Related Genes and Apoptosis-Related Genes, Respectively}

Ferroptosis-related genes were downloaded from FerrDb and apoptosis-related genes were downloaded from UniProt. We overlapped these genes with AD-related module genes derived from WGCNA, respectively. The

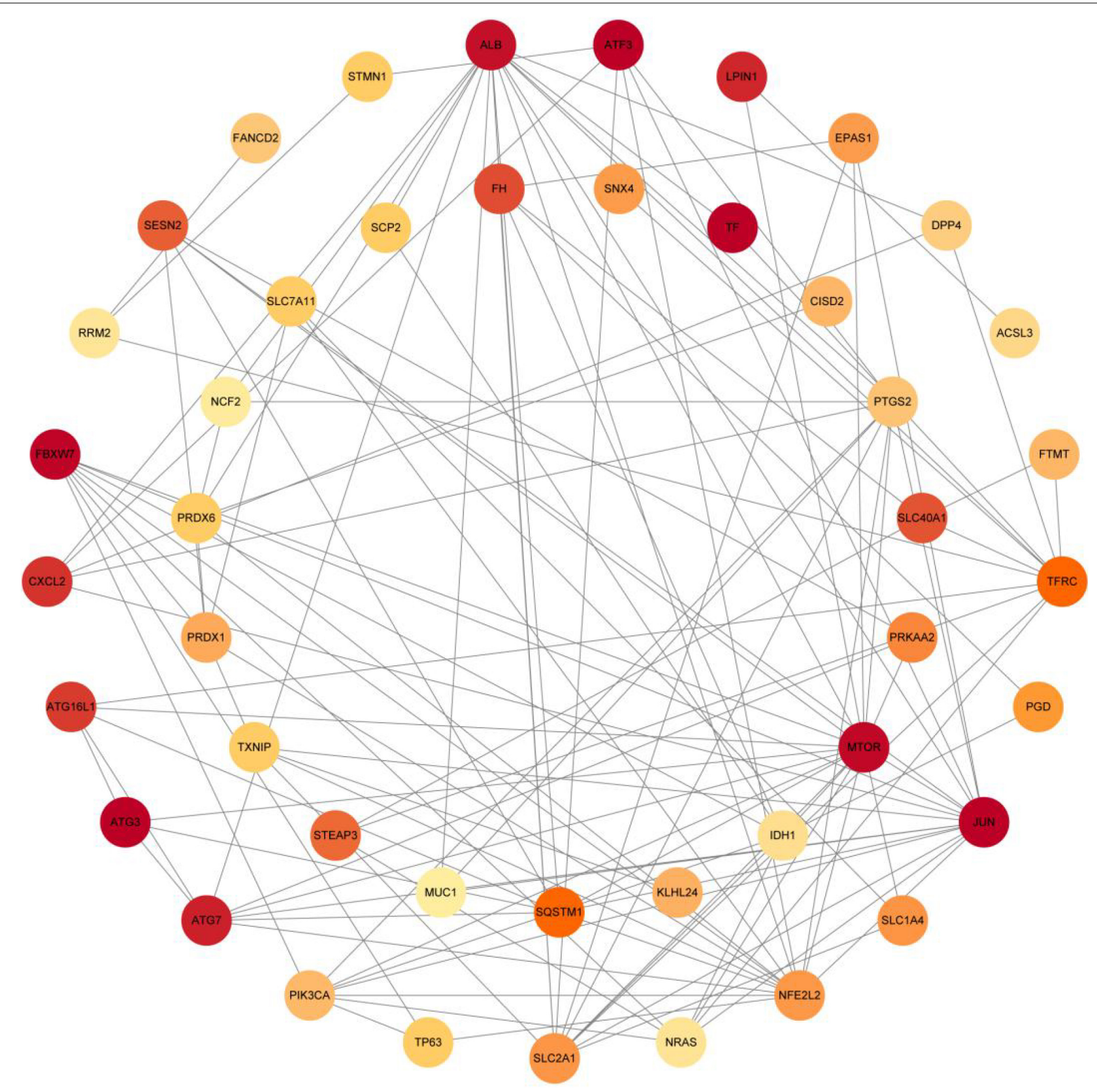

FIGURE 5 | Protein-protein interaction network of 53 ferroptosis-related module genes were analyzed using Cytoscape software. The network includes 44 nodes and 120 edges (The disconnected nodes were hided). The edges between 2 nodes represent the gene-gene interactions. The size and color of the nodes corresponding to each gene were determined according to the degree of interaction. Color gradients represent the variation of the degrees of each gene from high to low. 
Venn diagram was used to describe the details of the overlapped genes.

\section{Gene Ontology and Kyoto Encyclopedia of Genes and Genomes Enrichment Analysis of Overlapped Genes}

Functional enrichment analysis was performed in three domains of GO, including biological process (BP), cellular component (CC), and molecular function (MF). The KEGG database contains datasets of pathways involving biological functions, diseases, chemicals, and drugs. The enrichment analysis was carried out by clusterProfiler $\mathrm{R}$ package to determine the biological functions of the genes and associated pathways (Yu et al., 2012).

\section{Protein-Protein Interaction Establishment and Identification of Hub Genes}

An online tool (Search Tools for the Retrieval of Interacting Genes, STRING ${ }^{4}$ ) was used to analyze protein interactions. The PPI pairs were screened by confidence score $(>0.40)$, and the PPI network was visualized by the Cytoscape V3.9.0 software (Shannon et al., 2003). Three indicators (Degree, closeness, and Betweenness) were calculated through CytoHubba to evaluate the importance of each node, and the top 10 nodes were selected. The hub genes were their common nodes.

\section{Construction and Validation of the Logistic Regression}

To effectively differentiate the $\mathrm{AD}$ patients from controls, the logistic regression was constructed, and to evaluate the performance of the logistic regression model for predicting the occurrence of $\mathrm{AD}$, we performed receiver operating characteristic (ROC) curve analyses using the pROC package of $\mathrm{R}$ (Robin et al., 2011). We selected the statistically significant genes from hub genes $(P<0.05)$ and used the nomogram to predict the occurrence of AD. The expression level of the hub genes was shown by the violin plot.

\section{RESULTS}

\section{Weighted Co-expression Network Construction and Identification of Core Modules}

The scale-free network was constructed with the soft threshold set to $4\left(\mathrm{R}^{2}=0.905\right)$ (Figures $\left.2 \mathrm{~A}, \mathrm{~B}\right)$. Then, the adjacency matrix and topological overlap matrix were built. We then calculated the module eigengenes representing the overall gene expression level of each module; these were clustered based on their correlation. A total of 4 modules were identified and

${ }^{4}$ https://string-db.org/ labeled with a unique color (Figure 2C). We analyzed the correlations of each eigengene with phenotype (AD or control samples), and found two modules were correlated with $\mathrm{AD}$ namely, the turquoise (cor $=-0.32, P=2 \mathrm{e}-13$ ), and blue (cor $=0.30, P=1 \mathrm{e}-11$ ) modules (Figure 2D). The 4,617 genes in these modules-which are associated with $\mathrm{AD}$-were retained for further analysis.

\section{The Extraction of Ferroptosis-Related Genes From FerrDb and Apoptosis-Related Genes From UniProt}

The ferroptosis-related genes were downloaded and summarized from the FerrDb (Zhou and Bao, 2020; Table 1). 253 regulatory factors (including 108 drivers, 69 suppressors, 35 inducers, and 41 inhibitors), 111 markers, and 95 ferroptosis-related diseases were collated by FerrDb. We have extracted 2,130 genes from Uniprot, which is related to apoptosis.

\section{Overlap Alzheimer's Disease-Related Module Genes With Ferroptosis-Related Genes and Apoptosis-Related Genes, \\ Respectively}

We overlapped the AD-related module genes derived from WGCNA with ferroptosis-related genes extracted from FerrDb, 53 overlapped genes were obtained, namely ferroptosis-related module genes, which was shown by the Venn diagram (Figure 3A). The details of overlapped genes, including 19 drivers, 16 suppressors, and 18 markers, were shown in Table 2. We also overlapped the AD-related module genes with apoptosis-related genes to obtain apoptosis-related module genes as a control for further analysis, and 90 overlapped genes were obtained, which was also shown by the Venn diagram (Figure 3B).

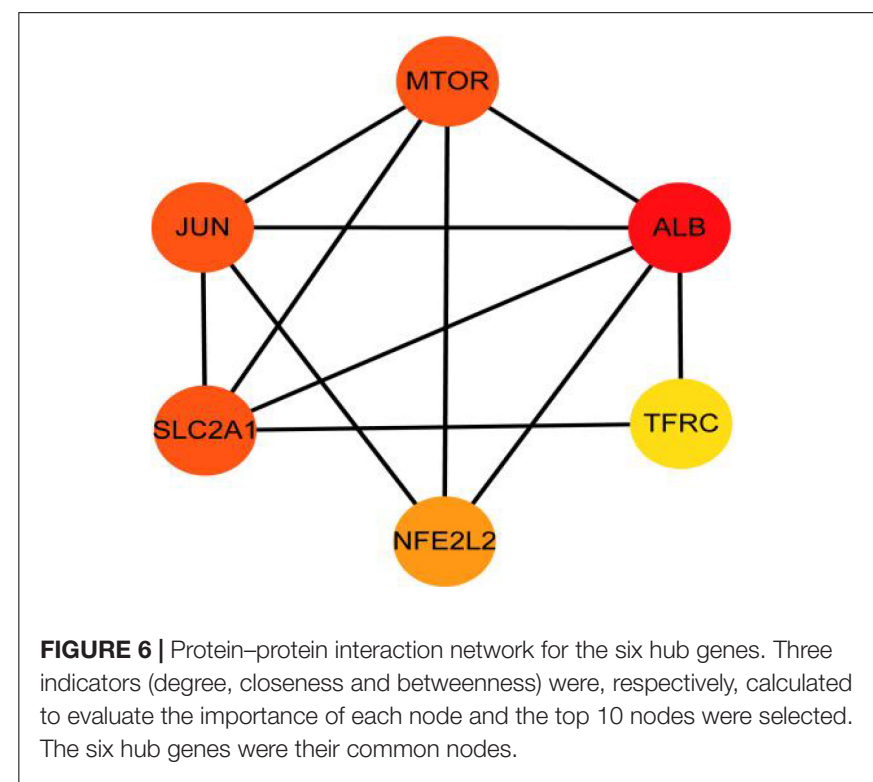




\section{Gene Ontology and Kyoto Encyclopedia of Genes and Genomes Enrichment Analysis of Overlapped Genes}

The significant GO functional terms of the 53 ferroptosisrelated module genes, including $\mathrm{BP}, \mathrm{MF}$, and $\mathrm{CC}$, were illustrated in Figure 4A. The significant terms of GO-BP were principally associated with the response to stress, such as the response to oxidative stress. The pathways enriched by GO-MF were principally associated with the activity of peroxidase, oxidoreductase, and antioxidant. The ferric iron-binding was also enriched by the GO-MF. The analysis of GO-CC indicated that overlapped genes were significantly enriched in basolateral plasma membrane, phagophore assembly site, pigment granule, and melanosome. The KEGG analysis showed that these overlapped genes were enriched not only in renal cell carcinoma pathways and central carbon metabolism in cancer, but also in autophagy-related pathways and ferroptosis (Figure 4B). The

A ROC curve for Logistic Regression model

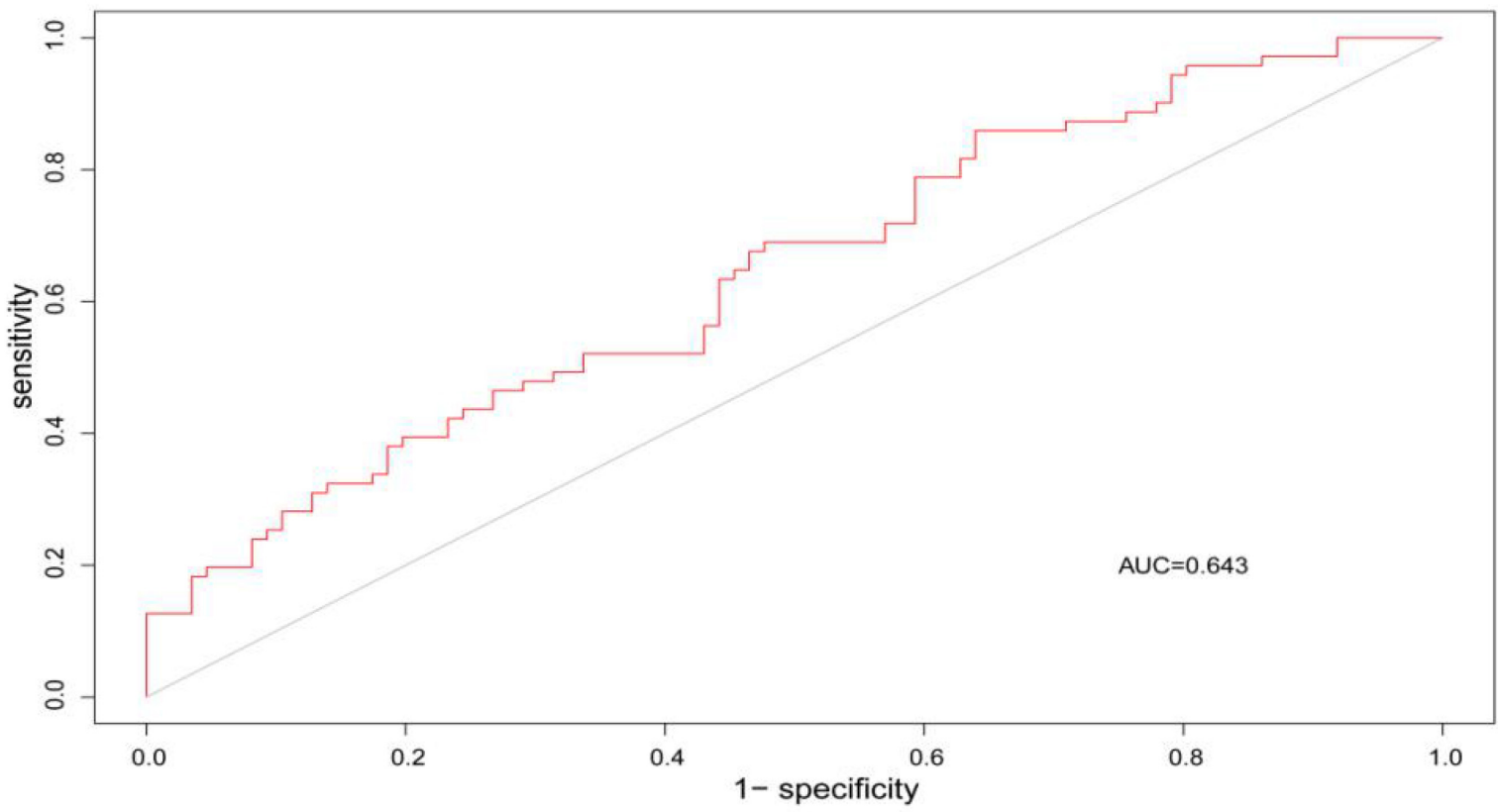

B

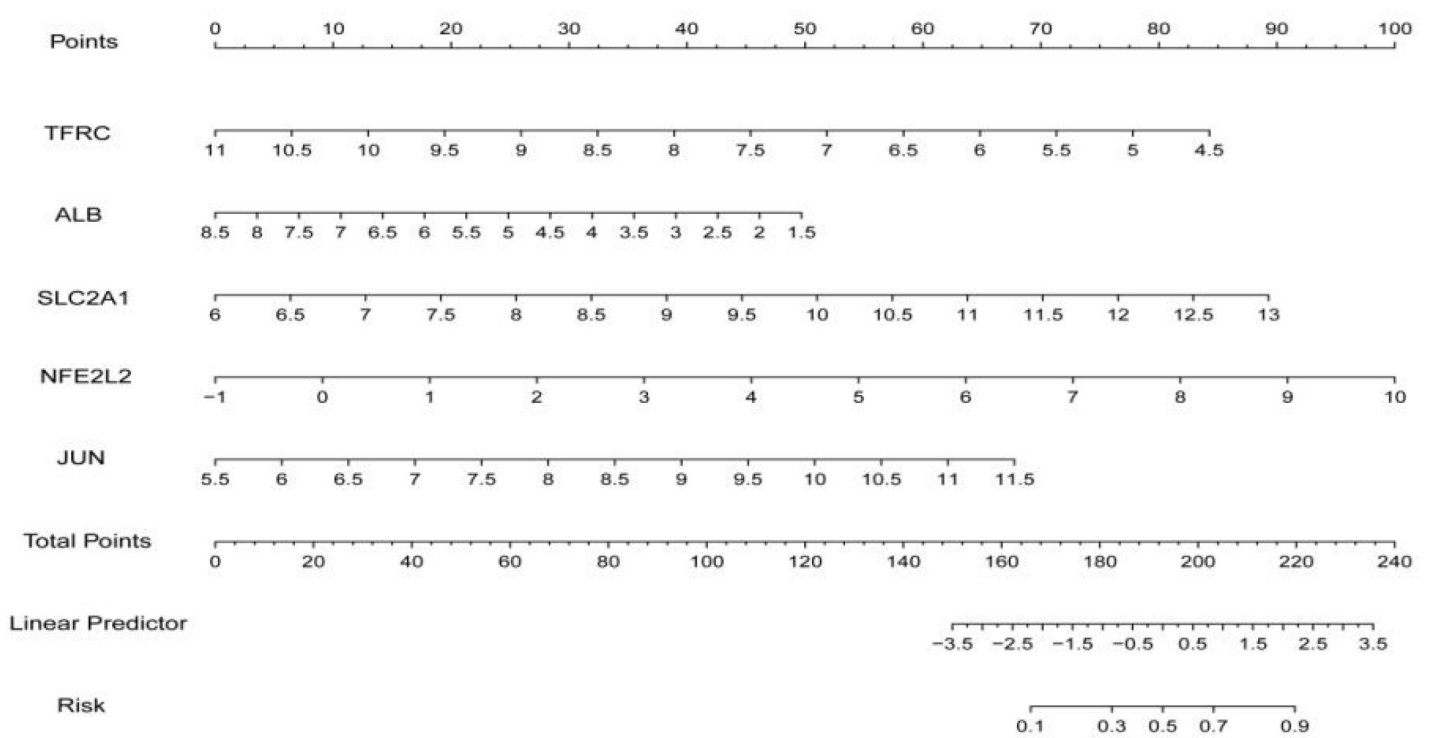

FIGURE 7 | (A) ROC curve was used to evaluate the performance of the logistic regression model. The area under the curve (AUC) was 0.643. (B) The nomogram was used to predict the occurrence of AD. Ferroptosis-related hub genes, JUN, SLC2A1, TFRC, ALB, and NFE2L2 (P < 0.05), were included in this nomogram. 
pathway of ferroptosis was enriched by KEGG, suggesting that these overlapped genes were significant for our study and could be used for further analysis.

\section{Protein-Protein Interaction Establishment and Identification of Hub Genes}

The PPI analysis of 53 ferroptosis-related module genes was performed through the STRING database and visualized by Cytoscape V3.9.0 (Figure 5). JUN, SLC2A1, TFRC, ALB, MTOR, and NFE2L2 were taken as potential hub genes based on Degree, closeness, and betweenness. The hub genes were their common top ten nodes. The PPI network of the hub genes was presented in Figure 6. Similarly, the identification of hub genes of apoptosis-related module genes was also conducted, and STAT1, CFLAR, FASLG, MCL1 and BCL2L11 were obtained from the 90 overlapped genes.

\section{Construction and Validation of the Logistic Regression}

Through constructing the logistic regression, JUN, SLC2A1, TFRC, ALB, and NFE2L2 were selected, which could effectively differentiate $\mathrm{AD}$ patients from controls $(P<0.05)$. The $\mathrm{P}$-value of MTOR was more than 0.05 , which was not statistically significant. We used the ROC curve to evaluate the performance of the logistic regression model (the area under the ROC curve of the model was 0.643 ), and the nomogram was used for predicting the occurrence of AD (Figures 7A,B). The expression level of the five hub genes is shown in Figure 8. Similarly, the logistic regression was also constructed for apoptosis-related hub genes, and STAT1, MCL1, and BCL2L11 were selected and could distinguish AD patients from controls $(\mathrm{P}<0.05)$. The AUC was 0.608 , which was less than the former AUC value, suggesting that ferroptosis was more special than apoptosis in AD. The ROC curve and nomogram are shown in Figures 9A,B.

\section{DISCUSSION}

The pathological process of ferroptosis has some characteristics in common with $\mathrm{AD}$, such as excess iron accumulation and elevated lipid peroxides. It has been reported that the pathological process of ferroptosis could be directly induced by iron overload (Wang et al., 2017; Fang et al., 2019). Clinically, lipid peroxidation metabolites were highly correlated with the progression of AD (Benseny-Cases et al., 2014). Besides, it has also been reported that reactive oxygen species (Wang et al., 2016) and reduced glutathione (Chiang et al., 2017) were found in the pathological process of $\mathrm{AD}$. However, how does ferroptosis mediate $\mathrm{AD}$ ? Some ferroptosis-related signaling pathways were found in $\mathrm{AD}$, such as iron metabolism pathway, redox homeostasis pathway,

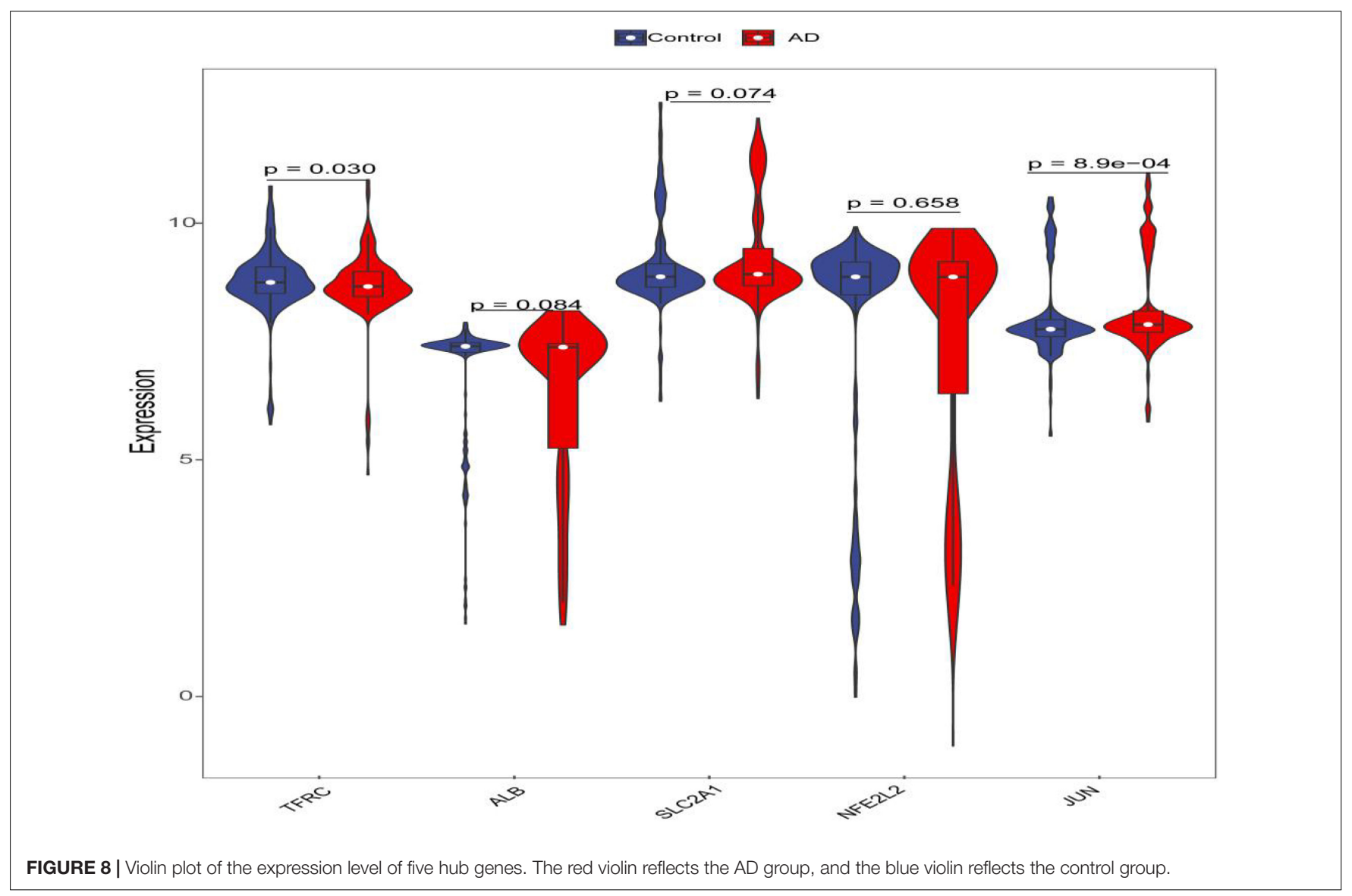


A ROC curve for Logistic Regression model

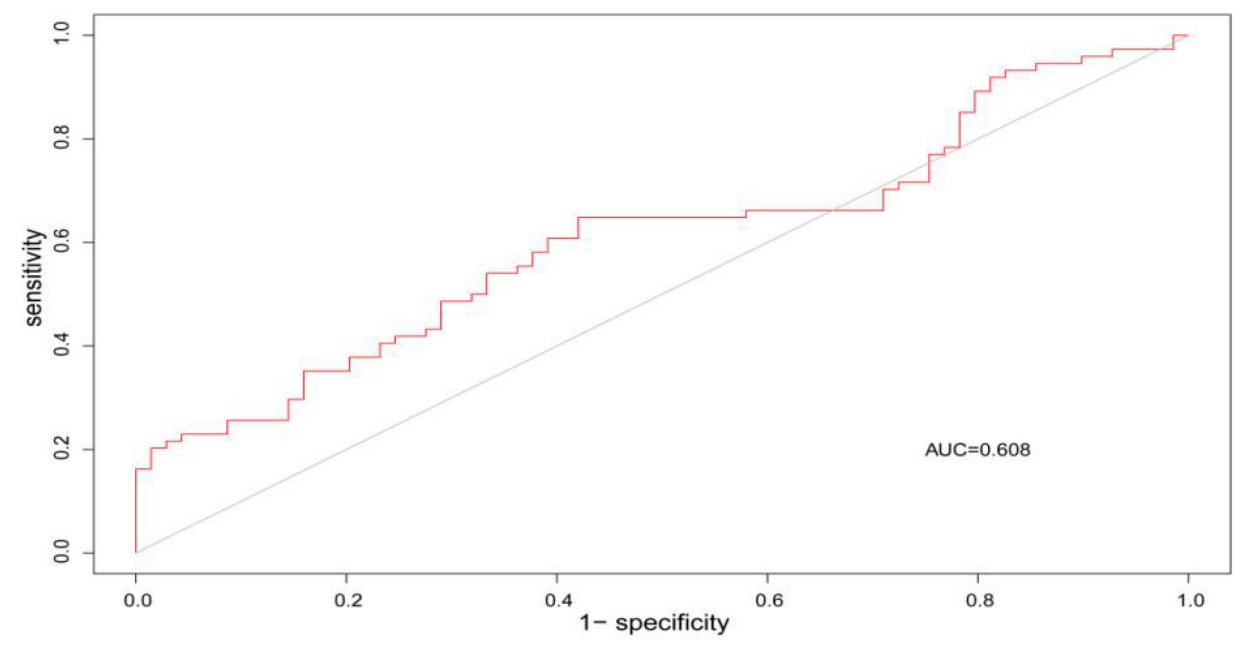

B

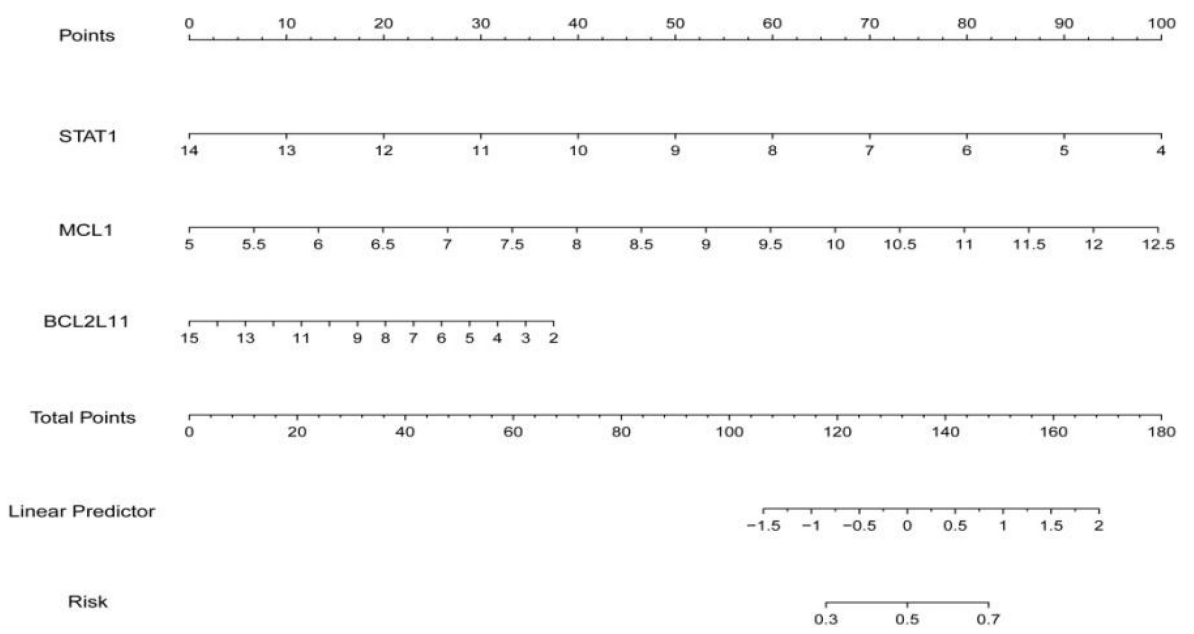

FIGURE 9 | (A) The area under the curve (AUC) was 0.608. (B) The nomogram of apoptosis-related hub genes, STAT1, MCL1, and BCL2L11 (P < 0.05).

and lipid metabolism pathway (Chen et al., 2021). Exploring of the mechanism of ferroptosis in $\mathrm{AD}$ could provide a novel therapeutic target for the treatment of $\mathrm{AD}$ and possibly, other

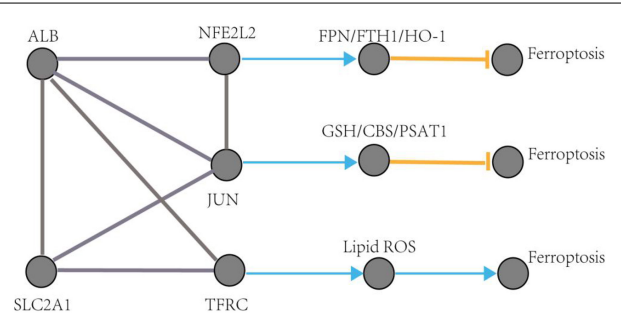

FIGURE 10 | The regulation pathways of JUN, SLC2A1, TFRC, ALB, and NFE2L2 participating in ferroptosis in AD. The gray edge represents the gene-gene interactions. The orange T-shaped edge denotes suppression, and the blue arrow denotes promotion. neurodegenerative diseases (Ashraf and So, 2020). This study identified five hub genes that may participate in the pathologic processes associated with ferroptosis in $\mathrm{AD}$. The possible pathways of these five genes involved in ferroptosis are shown in Figure 10 (see text footnote 2) (Gao et al., 2016; Shin et al., 2018; Chen et al., 2019).

Emerging evidence has demonstrated that ferroptosis could be a therapeutic target for AD (Gleason and Bush, 2021). Some ferroptosis inhibitors, such as iron-chelators and vitamin E, have shown clinical efficacy in treating AD. Deiprone is a brain osmotic iron-chelating agent currently in phase II clinical trials to treat AD (Nikseresht et al., 2019). Antioxidant vitamin E could delay decline in function and relieve caregiver burden in patients with AD (Dysken et al., 2014a,b). Collectively, Patients with $\mathrm{AD}$ may benefit from ferroptosis as a therapeutic target. Unlike targeting $\beta$-amyloid, the clinical trials of ferroptosis inhibitors are still in the exploratory stage and need to be dose-optimized and replicated on a larger scale (Nikseresht et al., 2019). The clinical 
efficacy of ferroptosis inhibitors in the treatment of $\mathrm{AD}$ also needs to be further improved.

There were some limitations to this study. Firstly, while selecting datasets for differentially expressed analysis, it was found that some datasets had fewer or no differentially expressed genes (DEGs, correcting $P$-value $<0.05$ and $|\operatorname{logFC}| \geq 1.0$ ), such as GSE48350 (Berchtold et al., 2013) and GSE131617 (Miyashita et al., 2014; Kikuchi et al., 2020). Therefore, the datasets and related $\mathrm{AD}$ patients we can choose are still limited. In addition, if the DEGs further overlaps with the ferroptosisrelated module genes, the number of available genes are limited and could not be used for further analysis. Secondly, the potential ferroptosis-related biomarkers identified by this study still need further literature support and laboratory evidence verification. Thirdly, the ferroptosis-related genes are derived from FerrDb, which is being updated continuously, and more genes are yet to be discovered.

\section{CONCLUSION}

We identified five hub genes (JUN, SLC2A1, TFRC, ALB, and NFE2L2) that are closely associated with ferroptosis in AD and can differentiate $\mathrm{AD}$ patients from controls, and are thus potential ferroptosis-related biomarkers for disease diagnosis and therapeutic monitoring. Three hub genes of apoptosis-related

\section{REFERENCES}

Ashraf, A., and So, P. W. (2020). Spotlight on Ferroptosis: iron-Dependent Cell Death in Alzheimer's Disease. Front. Aging Neurosci. 12:196. doi: 10.3389/fnagi. 2020.00196

Ayton, S., Wang, Y., Diouf, I., Schneider, J. A., Brockman, J., Morris, M. C., et al. (2019). Brain iron is associated with accelerated cognitive decline in people with Alzheimer pathology. Mol. Psychiatry 25, 2932-2941. doi: 10.1038/s41380-0190375-7

Benseny-Cases, N., Klementieva, O., Cotte, M., Ferrer, I., and Cladera, J. (2014). Microspectroscopy (muFTIR) reveals co-localization of lipid oxidation and amyloid plaques in human Alzheimer disease brains. Anal. Chem. 86, 1204712054. doi: 10.1021/ac502667b

Berchtold, N. C., Coleman, P. D., Cribbs, D. H., Rogers, J., Gillen, D. L., and Cotman, C. W. (2013). Synaptic genes are extensively downregulated across multiple brain regions in normal human aging and Alzheimer's disease. Neurobiol. Aging 34, 1653-1661. doi: 10.1016/j.neurobiolaging.2012.11.024

Chen, K., Jiang, X., Wu, M., Cao, X., Bao, W., and Zhu, L. Q. (2021). Ferroptosis, a Potential Therapeutic Target in Alzheimer's Disease. Front. Cell Dev. Biol. 9:704298. doi: 10.3389/fcell.2021.704298

Chen, Y., Zhu, G., Liu, Y., Wu, Q., Zhang, X., and Bian, Z. (2019). O-GlcNAcylated c-Jun antagonizes ferroptosis via inhibiting GSH synthesis in liver cancer. Cell Signal 63:109384. doi: 10.1016/j.cellsig.2019.109384

Chiang, G. C., Mao, X., Kang, G., Chang, E., Pandya, S., Vallabhajosula, S., et al. (2017). Relationships among cortical glutathione levels, brain amyloidosis, and memory in healthy older adults investigated in vivo with H-1-MRS and pittsburgh compound-B PET. Am. J. Neuroradiol. 38, 1130-1137. doi: 10.3174/ ajnr.a5143

Dysken, M. W., Guarino, P. D., Vertrees, J. E., Asthana, S., Sano, M., Llorente, M., et al. (2014a). Vitamin E and memantine in Alzheimer's disease: clinical trial methods and baseline data. Alzheimer's Dement. 10, 36-44. doi: 10.1016/j.jalz. 2013.01.014

Dysken, M. W., Sano, M., Asthana, S., Vertrees, J. E., Pallaki, M., Llorente, M., et al. (2014b). Effect of vitamin $\mathrm{E}$ and memantine on functional decline in Alzheimer disease: the TEAM-AD VA cooperative randomized trial. JAMA 311, 1161-1161. doi: 10.1001/jama.2013.282834 genes in AD (STAT1, MCL1, and BCL2L11) were also identified as a control to show the specificity of Ferroptosis. JUN, SLC2A1, TFRC, ALB, and NFE2L2 are thus potential ferroptosis-related biomarkers for disease diagnosis and therapeutic monitoring.

\section{DATA AVAILABILITY STATEMENT}

The datasets presented in this study can be found in online repositories. The names of the repository/repositories and accession number(s) can be found in the article/ supplementary material.

\section{AUTHOR CONTRIBUTIONS}

YW, GC, and WS contributed equally to this work. All authors contributed toward data analysis, drafted and critically revised the manuscript, gave final approval of the version to be published, and agreed to be accountable for all aspects of the work.

\section{ACKNOWLEDGMENTS}

We would like to thank the data support provided by the GEO databases, FerrDb and Uniprot.

Fang, X. X., Wang, H., Han, D., Xie, E. J., Yang, X., Wei, J. Y., et al. (2019). Ferroptosis as a target for protection against cardiomyopathy. Proc. Natl. Acad. Sci. US A. 116, 2672-2680. doi: 10.1073/pnas.1821022116

Gao, M., Monian, P., Pan, Q., Zhang, W., Xiang, J., and Jiang, X. (2016). Ferroptosis is an autophagic cell death process. Cell Res. 26, 1021-1032. doi: 10.1038/cr. 2016.95

Gbd 2016 Dementia Collaborators. (2019). Global, regional, and national burden of Alzheimer's disease and other dementias, 1990-2016: a systematic analysis for the Global Burden of Disease Study 2016. Lancet Neurol. 18, 88-106. doi: 10.1016/S1474-4422(18)30403-4

Gleason, A., and Bush, A. I. (2021). Iron and Ferroptosis as Therapeutic Targets in Alzheimer's Disease. Neurotherapeutics 18, 252-264. doi: 10.1007/sl3311-02000954-y

Hambright, W. S., Fonseca, R. S., Chen, L., Na, R., and Ran, Q. (2017). Ablation of ferroptosis regulator glutathione peroxidase 4 in forebrain neurons promotes cognitive impairment and neurodegeneration. Redox Biol. 12, 8-17. doi: 10. 1016/j.redox.2017.01.021

Jakaria, M., Bush, A. I., and Ayton, S. (2021). Ferroptosis as a mechanism of neurodegeneration in Alzheimer's disease. J. Neurochem. 159, 804-825. doi: 10.1111/jnc.15519

Kikuchi, M., Sekiya, M., Hara, N., Miyashita, A., Kuwano, R., Ikeuchi, T., et al. (2020). Disruption of a RAC1-centred network is associated with Alzheimer's disease pathology and causes age-dependent neurodegeneration. Hum. Mol. Genet. 29, 817-833. doi: 10.1093/hmg/ddz320

Lane, D. J. R., Ayton, S., and Bush, A. I. (2018). Iron and Alzheimer's Disease: an Update on Emerging Mechanisms. J. Alzheimers Dis. 64, S379-S395. doi: 10.3233/JAD- 179944

Langfelder, P., and Horvath, S. (2008). WGCNA: an R package for weighted correlation network analysis. BMC Bioinformatics 9:559. doi: 10.1186/14712105-559

Langfelder, P., and Horvath, S. (2012). Fast R Functions for Robust Correlations and Hierarchical Clustering. J. Statist. Softw. 46:11.

Masaldan, S., Bush, A. I., Devos, D., Rolland, A. S., and Moreau, C. (2019). Striking while the iron is hot: Iron metabolism and ferroptosis in neurodegeneration. Free Radic. Biol. Med. 133, 221-233. doi: 10.1016/j.freeradbiomed.2018.0 9.033 
Mckay, E. C., Beck, J. S., Khoo, S. K., Dykema, K. J., Cottingham, S. L., and Winn, M. E. (2019). Peri-Infarct Upregulation of the Oxytocin Receptor in Vascular Dementia. J. Neuropathol. Exp. Neurol. 78, 436-452. doi: 10.1093/jnen/nl z023

Miyashita, A., Hatsuta, H., Kikuchi, M., Nakaya, A., Saito, Y., Tsukie, T., et al. (2014). Genes associated with the progression of neurofibrillary tangles in Alzheimer's disease. Transl. Psychiatry 4:e396. doi: 10.1038/tp.2014.35

Nikseresht, S., Bush, A. I., and Ayton, S. (2019). Treating Alzheimer's disease by targeting iron. Br. J. Pharmacol. 176, 3622-3635. doi: 10.1111/bph.14567

Obulesu, M., and Lakshmi, M. J. (2014). Apoptosis in Alzheimer's disease: an understanding of the physiology, pathology and therapeutic avenues. Neurochem. Res. 39, 2301-2312. doi: 10.1007/s11064-014-1454-4

Plascencia-Villa, G., and Perry, G. (2021). Preventive and Therapeutic Strategies in Alzheimer's Disease: focus on Oxidative Stress. Antioxid. Redox Signal. 34, 591-610. doi: 10.1089/ars.2020.8134

Robin, X., Turck, N., Hainard, A., Tiberti, N., Lisacek, F., Sanchez, J. C., et al. (2011). pROC: an open-source package for R and S + to analyze and compare ROC curves. BMC Bioinform. 12:77. doi: 10.1186/1471-2105-12-77

Shannon, P., Markiel, A., Ozier, O., Baliga, N. S., Wang, J. T., Ramage, D., et al. (2003). Cytoscape: a software environment for integrated models of biomolecular interaction networks. Genome Res. 13, 2498-2504. doi: 10.1101/ gr.1239303

Sharma, V. K., Singh, T. G., Singh, S., Garg, N., and Dhiman, S. (2021). Apoptotic Pathways and Alzheimer's Disease: probing Therapeutic Potential. Neurochem. Res. 46, 3103-3122. doi: 10.1007/s11064-021-03418-7

Shimohama, S. (2000). Apoptosis in Alzheimer's disease-an update. Apoptosis 5, 9-16. doi: 10.1023/a:1009625323388

Shin, D., Kim, E. H., Lee, J., and Roh, J. L. (2018). Nrf2 inhibition reverses resistance to GPX4 inhibitor-induced ferroptosis in head and neck cancer. Free Radi. Biol. Med. 129, 454-462. doi: 10.1016/j.freeradbiomed.20180.10.426

Tan, M. G., Chua, W. T., Esiri, M. M., Smith, A. D., Vinters, H. V., and Lai, M. K. (2010). Genome wide profiling of altered gene expression in the neocortex of Alzheimer's disease. J. Neurosci. Res. 88, 1157-1169. doi: 10.1002/jnr.22290

Vitalakumar, D., Sharma, A., and Flora, S. J. S. (2021). Ferroptosis: a potential therapeutic target for neurodegenerative diseases. J. Biochem. Mol. Toxicol. 35:e22830. doi: 10.1002/jbt.22830

Wang, H., An, P., Xie, E., Wu, Q., Fang, X., Gao, H., et al. (2017). Characterization of ferroptosis in murine models of hemochromatosis. Hepatology 66, 449-465. doi: 10.1002/hep.29117
Wang, X., Hu, X., Yang, Y., Takata, T., and Sakurai, T. (2016). Nicotinamide mononucleotide protects against $\beta$-amyloid oligomer-induced cognitive impairment and neuronal death. Brain Res. 1643, 1-9. doi: 10.1016/j.brainres. 2016.04.060

Weiland, A., Wang, Y., Wu, W., Lan, X., Han, X., Li, Q., et al. (2019). Ferroptosis and Its Role in Diverse Brain Diseases. Mol. Neurobiol. 56, 4880-4893. doi: 10.1007/s12035-018-1403-3

Yan, N., and Zhang, J. (2019). Iron Metabolism. Front. Neurosci. 13:1443. doi: 10.3389/fnins.2019.01443

Yu, G., Wang, L. G., Han, Y., and He, Q. Y. (2012). clusterProfiler: an R package for comparing biological themes among gene clusters. OMICS 16, 284-287. doi: $10.1089 /$ omi.2011.0118

Zhang, C., Rodriguez, C., Spaulding, J., Aw, T. Y., and Feng, J. (2012). Agedependent and tissue-related glutathione redox status in a mouse model of Alzheimer's disease. J. Alzheimers Dis. 28, 655-666. doi: 10.3233/JAD-2011111244

Zhang, G., Zhang, Y., Shen, Y., Wang, Y., Zhao, M., and Sun, L. (2021). The Potential Role of Ferroptosis in Alzheimer's Disease. J. Alzheimers Dis. 80, 907-925. doi: 10.3233/JAD-201369

Zhou, N., and Bao, J. (2020). FerrDb: a manually curated resource for regulators and markers of ferroptosis and ferroptosis-disease associations. Database J. Biol. Databases Curation 2020:baaa021. doi: 10.1093/database/baaa021

Conflict of Interest: The authors declare that the research was conducted in the absence of any commercial or financial relationships that could be construed as a potential conflict of interest.

Publisher's Note: All claims expressed in this article are solely those of the authors and do not necessarily represent those of their affiliated organizations, or those of the publisher, the editors and the reviewers. Any product that may be evaluated in this article, or claim that may be made by its manufacturer, is not guaranteed or endorsed by the publisher.

Copyright (c) 2022 Wang, Chen and Shao. This is an open-access article distributed under the terms of the Creative Commons Attribution License (CC BY). The use, distribution or reproduction in other forums is permitted, provided the original author(s) and the copyright owner(s) are credited and that the original publication in this journal is cited, in accordance with accepted academic practice. No use, distribution or reproduction is permitted which does not comply with these terms. 